\title{
Endometrial adenocarcinoma: An analysis of treatment and outcome
}

\author{
LOUISE M. BYRD ${ }^{1}$, RICHARD SWINDELL ${ }^{2}$, DANIEL WEBBER-ROOKES ${ }^{2}$, \\ R. HANNON ${ }^{2}$, ROBIN D. HUNTER ${ }^{2}$, JAC LIVSEY $^{2}$ and SUSAN E. DAVIDSON ${ }^{2}$ \\ ${ }^{1}$ St Mary's Hospital for Women and Children, Hathersage Road, Whitworth Park, Manchester M13 9WL; \\ ${ }^{2}$ Paterson Institute for Cancer Research: Department of Clinical Oncology: Department of Medical \\ Statistics: Christie Hospital NHS Trust, Wilmslow Road, Manchester M20 9BX, UK
}

Received March 7, 2008; Accepted May 5, 2008

DOI: 10.3892/or_00000133

\begin{abstract}
This study aims to review the survival and morbidity in patients treated for endometrial cancer, at a single centre and analyses the effects of co-morbidity on these outcomes. Case notes of all patients referred to the Christie Hospital with endometrial carcinoma from January 1, 1993 to December 31, $1995(n=499)$ were reviewed. Twenty patients presented with recurrence and were not included in this analysis. Three hundred and seventy-five patients had previously undergone a total abdominal hysterectomy and bilateral salpingoophorectomy (+/- pelvic lymphadenectomy). Of these, 175 received adjuvant external beam radiotherapy (XRT) only, 49 received XRT and brachytherapy, 30 received brachytherapy alone and 121 patients had no further therapy. One hundred and four patients were referred for primary treatment. Radical radiotherapy was administered to 63 patients who were unfit for surgery, with 10 of these receiving $\mathrm{XRT}+$ brachytherapy and 53 receiving brachytherapy alone. Thirteen patients received palliative XRT and 28 supportive care only. The overall 5-year survival for those treated radically was $73.3 \%$. There was no significant survival difference between patients who underwent surgery and adjuvant radiotherapy, in whatever form $(\mathrm{p}=0.115)$. Patients who did not undergo surgery did less well as a group, although there was no significant survival difference between those treated with combination therapy or brachytherapy alone $(\mathrm{p}=0.33)$. Survival was significantly associated with FIGO stage, tumour grade, age (especially those $>75$ years) and co-morbidity (ACE-27 score). Late morbidity occurred in 46 patients, with severe toxicity affecting $12(3.8 \%)$. Toxicity was associated with
\end{abstract}

Correspondence to: Dr Louise Byrd, Department of Obstetrics and Gynaecology, St Mary's Hospital, Hathersage Road, Manchester M13 9WL, UK

E-mail: louise.byrd@cmmc.nhs.uk

Key words: endometrial adenocarcinoma, treatment, outcome, survival, co-morbidity
ACE-27 score ( $\mathrm{p}=0.0019)$, treatment dose and modality, with $50 \%(n=6)$ of severe toxicity seen in patients receiving adjuvant XRT + ICT. These data demonstrate that survival in patients with endometrial carcinoma treated radically remains good, with the stage and grade of tumour being significant factors for overall survival. The incidence of severe morbidity related to radiotherapy of any modality was $3.8 \%$. A high co-morbidity (ACE-27) score was significantly associated with poorer survival $(\mathrm{p}<0.0055)$ and increased late treatment morbidity $(\mathrm{p}=0.0019)$.

\section{Introduction}

Endometrial carcinoma is the most common malignancy of the female genital tract, with an incidence, in western countries, of 15-20/100,000 women per year (1). It is typically a cancer of post-menopausal women, with a peak incidence between 55 and 70 years of age, and a median age at diagnosis of 67 years.

Surgery is the cornerstone of treatment, consisting of total abdominal hysterectomy, with bilateral salpingooophorectomy. Pelvic sampling may be performed for early stage tumours with risk factors. Formal lymphadenectomy is reserved for more advanced disease (Stage II and above), though the role of this is controversial (ASTEC trial, unpublished data). If risk factors, including myometrial invasion up to and $>50 \%$ of the myometrial width and high-grade histology are identified, adjuvant radiotherapy, in the form of external beam therapy and/or brachytherapy to the vaginal vault, is usually given to reduce the risk of locoregional relapse. The value of postoperative radiotherapy is, however, controversial due to the lack of data from randomised controlled trials, the low relapse rate $(4-8 \%)$ seen in endometrial carcinoma and its limited, if any, impact on survival (2).

The morbidity of pelvic radiotherapy should not be underestimated. Whilst the rate of serious complications is low, a number of patients report long-term symptoms, which influence quality of life (3). This toxicity is particularly important as these patients have good long-term survival.

In recent years a larger proportion of patients are deemed unsuitable for radical surgery by virtue of severe co-morbid conditions, often cardiovascular or gross obesity. These patients can be treated successfully with primary radiotherapy (4). 
In addition, patients with locally advanced disease that is surgically inoperable may benefit from radio-therapy, given with radical or palliative intent.

\section{Materials and methods}

All patients, with a confirmed histological diagnosis of endometrial carcinoma, attending the Christie Hospital between January 1, 1993 and December 31, 1995 were identified and their records reviewed. The cohort comprised of 499 patients. Of these, 20 patients were referred at the time of recurrence and this group was considered separately and excluded from the mortality and morbidity analyses.

Treatment details. There are two main treatment groups: i) Patients treated with adjuvant radiotherapy following surgery due to high-risk prognostic factors (including myometrial invasion $>50 \%$ of the myometrial width in 156 patients, cervical extension in 24 and positive pelvic nodes in 2). Adjuvant treatment was external beam radiotherapy (XRT), XRT plus brachytherapy or brachytherapy alone. A total of 175 patients received post-operative adjuvant XRT, a further 49 received brachytherapy in addition to XRT, and 30, who were considered frail and not fit enough for XRT, received brachytherapy alone, and ii) Patients, considered unfit for surgery (by the gynaecological oncology surgeon or anaesthetist), were treated with primary radiotherapy. This comprised of 63 patients, 10 of whom received XRT plus brachytherapy for locally advanced disease and 53 who received brachytherapy alone, for disease thought to be confined to the uterus. However, this could not be confirmed by imaging in all cases, because of gross obesity.

In addition to these two groups, 13 patients were given XRT alone, with palliative intent. As they were deemed to be at low risk of recurrence, 121 patients received no adjuvant therapy. A further 28 patients with very poor performance status received supportive care only.

External beam radiotherapy (XRT) is summarised in Table I. The majority $(n=220)$ received a 4-field isocentric technique with megavoltage photons. This was given in 16 fractions to 196 patients, in 18 fractions to 2 patients, in 20 fractions to 21 patients and in 22 fractions to a further patient. The remaining 27 patients received a variable number of fractions, ranging from 4 to 15 . The majority of patients received either $4000(n=166)$ or $4250 \mathrm{cGy}(n=36)$. The remaining 44 patients received a variable dose (range 2000$3990 \mathrm{cGy}$ ). One additional patient received a total dose of 4500 cGy.

Brachytherapy was delivered, in all cases $(n=142)$, with low-dose rate caesium $\left(\mathrm{Cs}^{137}\right)$ using the Manchester system (4) with a single intrauterine tube, where the uterus was present, and vaginal ovoids. The dose was prescribed to the nominal body dose to give 6500 cGy in 2 insertions $(n=21)$. Those deemed unfit for more than one anaesthetic received 4000 cGy in one insertion $(n=32)$. Following hysterectomy, caesium vaginal ovoids were used alone, prescribing to the Manchester point A (5), to give a 48-h radium equivalent to 49 patients after external beam therapy, and a 96-h radium equivalent to 30 patients treated with vault caesium alone after surgery.
Patients were reviewed 6 weeks post-radiotherapy, every 4 months for the first 2 years and then every 6 months for the 5-year post-treatment. Patients receiving brachytherapy were given a vaginal dilator with instructions for use to try and prevent vaginal stenosis.

Treatment morbidity data were recorded from the notes using the Franco-Italian glossary (5).

Co-morbidities are defined as diseases, illnesses and/or conditions affecting a patient, but unrelated to the index cancer. Taxonomies for classifying such co-morbidity have been constructed, and include the Charlson co-morbidity index (6), the cumulative illness rating scale (7) and the index of coexistent disease (8). Nevertheless, none of these have been measured in the context of carcinoma. The adult co-morbidity evaluation-27, or ACE, modified from the Kaplan-Feinstein index (KFI) which was developed for diabetic patients (9), has, however, been specifically designed to be used in this context, and it was recommended that it be included in the UK national cancer database (Bang D, et al, ASCO: Annual Meeting of the American Society of Clinical Oncology, May 2000). A comprehensive scoring system, ACE includes several conditions such as dementia and AIDS, which have hitherto been overlooked in previous ratings, containing 27 items in total. Within the ACE score, disease states are ranked from zero to three, based on severity of the condition and the prognostic impact.

Following the classification of each co-morbid condition, each patient is given an overall ACE-27 score, based on the highest-ranked individual ailment. When two or more conditions, in different organ systems, were ranked as moderate (score 2), the overall ACE-27 is recorded as severe (score 3). Patients were given an ACE score by two out of three people (R.H., D.W.R. and S.E.D.). This study was part of a pilot in the UK where ACE-27 was investigated in cancer patients as seen on the NHSIA website: http://www.icservices.nhs.uk/ cancer/pages/dataset/docs/cdp_lessons_learned_comorbidity _v51a.rtf.

Statistical methods. Disease-specific survival was defined as the time of registration at the hospital to the time the patient succumbed to endometrial cancer. The short survival of patients from an intercurrent cause had their survival times censored at the point of succumbing, i.e. they were not counted as cancer deaths. Survival figures have been calculated using the Kaplan-Meier method and compared using the log-rank test (10). Late morbidity was recorded if noted to occur after 90 days from the time of registration. Morbidity data were calculated again using Kaplan-Meier, and compared using the log-rank test. Data were analysed using SPSS (statistical package for Social Sciences) version 11.5. The Chi-square test was used to assess association. Tests were deemed to be statistically significant at $\mathrm{p} \leq 0.05$.

\section{Results}

Demographics. The mean age of patients was 66.1 years (range 31-93). The histological type, stage and grade of tumour are given in Tables II-IV.

The most frequent histological subtype was adenocarcinoma and was found in $81.8 \%$ of patients. The majority 
Table I. External beam radiotherapy (XRT) [given either alone or in combination with surgery (S) and/or brachytherapy (ICT)].

\begin{tabular}{lrrrrr}
\hline Treatment/technique & XRT & S+XRT & XRT+ICT & S+XRT+ICT & Total \\
\hline 4-field brick & 3 & 164 & 9 & 44 & 220 \\
Hexagonal (4-field) & 0 & 4 & 0 & 2 & 6 \\
Parallel pair & 10 & 6 & 1 & 2 & 19 \\
Other & 0 & 1 & 0 & 0 & 1 \\
Unknown & 0 & 0 & 0 & 1 & 1 \\
Total & 13 & 175 & 10 & 49 & 247 \\
\hline
\end{tabular}

Table II. Histological type.

\begin{tabular}{lcr}
\hline Histology & Number of patients & $\%$ \\
\hline Squamous cell (SCC) & 5 & 1.0 \\
Adenocarcinoma & 392 & 81.8 \\
Adenosquamous cell & 12 & 2.5 \\
Other & 32 & 6.7 \\
Unknown & 38 & 7.9 \\
Total & 479 & 100.0 \\
\hline
\end{tabular}

Table III. FIGO stage of disease.

\begin{tabular}{lcr}
\hline Stage & Number of patients & $\%$ \\
\hline I & 275 & 57.4 \\
II & 66 & 13.8 \\
III & 59 & 12.3 \\
IV & 15 & 3.1 \\
Unknown & 64 & 13.4 \\
Total & 479 & 100.0 \\
\hline
\end{tabular}

Table IV. Grade of disease.

\begin{tabular}{lcr}
\hline Grade & Number of patients & $\%$ \\
\hline Well differentiated (1) & 126 & 26.3 \\
Moderately differentiated (2) & 56 & 11.7 \\
Poorly differentiated & 76 & 15.9 \\
Grade not documented/given & 221 & 46.1 \\
Total & 479 & 100.0 \\
\hline
\end{tabular}

of patients presented with FIGO stage I/II disease (71.2\%), (Table III). In 64 patients, the tumour stage was unknown or not recorded. This reflects the inability to stage some of these patients adequately, particularly obese patients, as the mechanical beds in the scanners have weight limits and size restrictions. Examination under anaesthetic may also have been omitted in these patients because of risk.

Where the tumour grade was recorded (Table IV), 126 (26\%) tumours were well-differentiated.

Ace-27 (co-morbidity) scores. An ACE-27 score was calculated for a total of 449 patients. Two hundred and thirty-eight patients $(53 \%)$ scored 0, $129(29 \%)$ scored 1, 64 (14\%) scored 2 and 18 (4\%) patients scored 3.

Survival data. For the survival data, the 20 patients treated at the time of recurrence were excluded as this group was separate (see below) and follow-up time was too short for late morbidity assessment. The 5-year survival in this group $(\mathrm{n}=20)$ was $53.6 \%$.

The 5-year survival for 438 patients (excluding 28 patients who received supportive care only and 13 patients treated with palliative intent) was $73.3 \%$. There were 48 intercurrent cases of patients who succumbed (Table V), a further 18 where the cause of death was not known and one that was treatmentrelated (see later).

Survival was calculated for each treatment group and this is shown in Fig. 1.

The group treated with surgery and post-operative XRT $(\mathrm{n}=175)$ had a 5-year survival of $79 \%$ and the group receiving surgery, XRT and brachytherapy $(n=49)$ had a 5-year survival of $77 \%$. Those treated with surgery and brachytherapy $(n=30)$ fared as well as those who had surgery alone $(n=121)$, both with a 5-year survival of $70 \%$. Survival data were not significantly different between the radical treatment groups $(\mathrm{p}=0.115)$ which had surgery $+/$ - radiotherapy. Patients who did not receive surgery did less well. The group treated radically with brachytherapy alone $(n=53)$ had a 5-year survival of $66 \%$, whilst those treated radically with XRT and brachytherapy $(n=10)$ had a 5-year survival of $48 \%$. The observed survival difference was not statistically significant $(\mathrm{p}=0.33)$. Those patients treated with palliative radiotherapy had a 5-year survival of $23 \%$, similar to the $28 \%$ 5-year survival noted amongst those patients who received supportive care.

The ACE score correlated with the treatment modality. A significant number of patients with a score of 3 were amongst those who received no treatment $(25 \%)$ and/or radiotherapy alone $(13 \%)$. In contrast, the vast majority of patients who underwent surgery, either alone or in combination with radiotherapy had lower ACE scores. 


\section{Survival by Treatment}

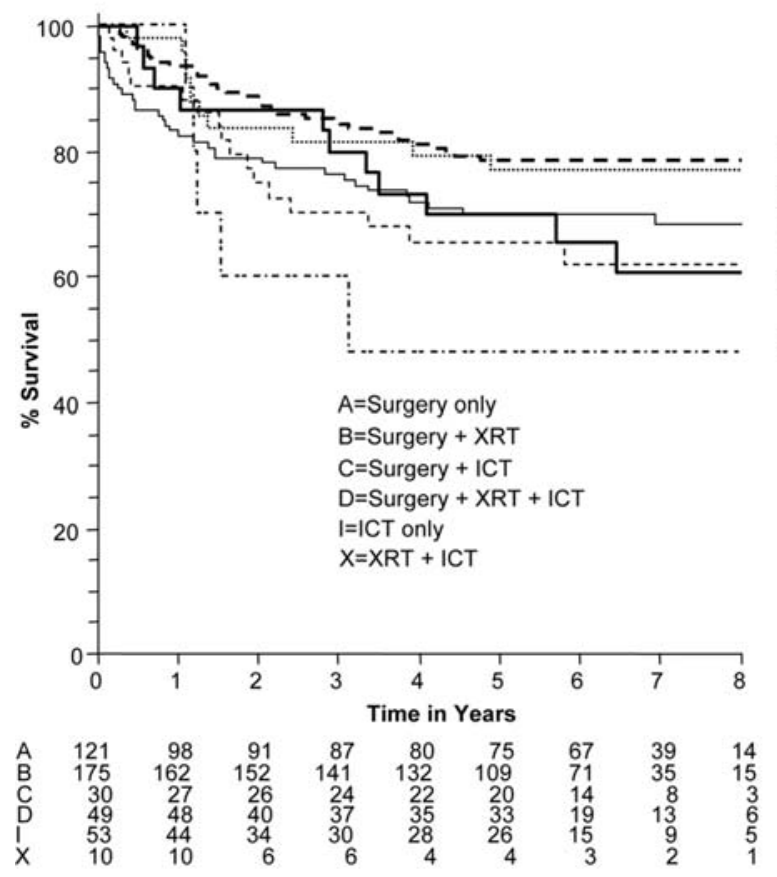

Figure 1. Survival according to the treatment received.
Table V. Number and cause of intercurrent deaths $(n=48)$.

\begin{tabular}{lc}
\hline Cause of death & Number of patients \\
\hline Myocardial infarction & 5 \\
Stroke & 3 \\
Heart failure & 6 \\
Pneumonia & 6 \\
Other & 4 \\
Unknown & 24 \\
Total & 48 \\
\hline
\end{tabular}

Table VI. Site of disease relapse $(n=45)$.

\begin{tabular}{lc}
\hline Site of relapse & Number of patients \\
\hline Central & 6 \\
Side wall & 1 \\
Distant & 33 \\
Central/distant & 2 \\
Side wall/distant & 3 \\
Total & 45 \\
\hline
\end{tabular}

$23(36 \%)$ had an ACE-27 score of 2 or 3, which reflects difficulties in imaging (because of obesity) and/or examination under anaesthesia (because of anaesthetic risk).

Site of relapse data. Relapse following treatment occurred in 45 out of 479 patients $(9.4 \%)$. The site of relapse is shown in Table VI and was cross-tabulated with treatment received in Table VII.

The most common site of relapse was distant metastases Distant metastases occurred most frequently in those receiving surgery and external beam radiotherapy (18/45), or all three modalities $(S+X+I)(6 / 45)$.

Treatment morbidity. Acute toxicity was poorly documented. Two patients had their treatment stopped at 18 fractions due to increased acute bowel toxicity.

Table VII. Site of disease relapse with treatment modality.

\begin{tabular}{|c|c|c|c|c|c|c|c|c|c|}
\hline Site/treatment & None & $\mathrm{S}$ & $\mathrm{X}$ & $\mathrm{S}+\mathrm{X}$ & I & $\mathrm{S}+\mathrm{I}$ & $\mathrm{X}+\mathrm{I}$ & $\mathrm{S}+\mathrm{X}+\mathrm{I}$ & Total \\
\hline None & 28 & 117 & 11 & 154 & 49 & 25 & 8 & 42 & 434 \\
\hline Central & 0 & 0 & 1 & 1 & 1 & 2 & 1 & 0 & 6 \\
\hline Side wall & 0 & 0 & 0 & 0 & 1 & 0 & 0 & 0 & 1 \\
\hline Distant & 0 & 3 & 1 & 18 & 2 & 2 & 1 & 6 & 33 \\
\hline Central/distant & 0 & 1 & 0 & 1 & 0 & 0 & 0 & 0 & 2 \\
\hline Side/distant & 0 & 0 & 0 & 1 & 0 & 1 & 0 & 1 & 3 \\
\hline Total & 28 & 121 & 13 & 175 & 53 & 30 & 10 & 49 & 479 \\
\hline
\end{tabular}

S, surgery; X, XRT and I, brachytherapy. 
Table VIII. Mild/moderate (grade ) morbidity following radiotherapy.

\begin{tabular}{|c|c|c|c|}
\hline $\begin{array}{l}\text { Patient } \\
\text { number }\end{array}$ & Bladder & Bowel & Vaginal \\
\hline
\end{tabular}

$\begin{array}{lll}1 & 1 \mathrm{C} \text { Bladder } & \\ 2 & & 1 \mathrm{~B} \text { Rectum } \\ 3 & & 1 \mathrm{~B} \text { Rectum } \\ 4 * & 2 \mathrm{C} \text { Bladder } & \\ 5 & & 1 \mathrm{~B} \text { Rectum } \\ 6 & \text { 1B Bladder } & 1 \mathrm{~B} \text { Rectum }\end{array}$

7

8

2C Bladder

2E Bladder

2B Rectum

1B Rectum

1B Vagina

1B Vagina

1B Vagina

1B Vagina

1B Vagina

1B Vagina

1B Vagina

1B Vagina

1B Vagina

1C Small bowel

1B Non-specific

1B Vagina

1B Vagina

1B Non-specific

2A Vagina

1C Bladder

1B Non-specific

IC Small bowel

2A Sigmoid

$1 \mathrm{C}$ Small bowel

2B Rectum

Those patients marked with * appear in both Table VIII and IX.

A total of 46 patients were recorded as having some late morbidity. Of these, 34 experienced grade $1 / 2$ morbidity (separate toxicities of 39, shown in Table VIII), whilst 12 (3.9\%) patients experienced grade 3/4 (shown in Table IX).

One patient succumbed during treatment (grade 4 morbidity) from peritonitis secondary to bowel perforation, which occurred 3 months following therapy. Unfortunately, this patient delayed in disclosing any symptoms until very unwell. Of the 11 patients reported as experiencing grade 3 morbidity, more than one organ was affected in three cases (total number of reported toxicities of 16). Five of these had complete vaginal stenosis. Four patients $(7.5 \%)$ receiving treatment by brachytherapy alone experienced severe (grade 3 ) morbidity, whilst 6 patients $(10 \%)$ receiving treatment with a combination of XRT and brachytherapy (with or without surgery), reported grade $3 / 4$ morbidity. The incidence of severe morbidity related to the radiotherapy of any modality was $3.8 \%$

On the univariate analysis, the ACE score was associated with late morbidity with $\mathrm{p}=0.0019$.

Though associated with survival, age did not influence morbidity in any of the age bands $(<55,<65,<75$ and $>75$ years $)(\mathrm{p}=0.51)$.

Histology, stage and grade did not appear to be significantly associated with morbidity $(\mathrm{p}=0.16 ; 0.698$ and 0.0598 , respectively).

The administered treatment dose was highly significant when it came to severe (grade $3 / 4$ ) morbidity ( $\mathrm{p}=0.0111$ ). This, in turn correlated with the number of fractions of radiotherapy $(p=0.0022)$ and number of insertions $(p<0.00005)$. On the multivariate analysis the number of insertions remained the most significant influence on morbidity $(\mathrm{p}<0.00005)$.

Finally, by using the Chi-square test we were able to demonstrate an association between the treatment received and the age and/or ACE-27 score. A greater proportion of patients $>75$ years, and/or with ACE-27 score of 3, received either no treatment or ICT as sole therapy. Conversely, this same group were much less likely to receive adjuvant radiotherapy, and slightly less likely to receive combination $(\mathrm{S}+\mathrm{X}+\mathrm{I})$ therapy.

\section{Discussion}

Based on the FIGO annual report (11) $76.5 \%$ of endometrial cancer patients are alive at 5 years, as the majority of these cancers are diagnosed at an early stage $(75-80 \%$, Stage I). This is comparable to the results of this data set where $71.2 \%$ of patients had Stage I/II disease, and the 5-year survival of those patients treated with curative intent was $73.3 \%$.

Results published in 2003, of a study of 181 patients with endometrial cancer recruited over a 10 -year period aiming to evaluate prognostic factors, demonstrated by multivariate analysis that overall survival correlated directly with FIGO stage, tumour grading, tumour type, depth of myometrial invasion and progesterone receptor status (12). We report similar findings with regard to the stage $(\mathrm{p}<0.0005)$ and grade $(\mathrm{p}=0.0006)$ of tumour, although the histological subtype was not significant $(\mathrm{p}=0.448)$ in this series.

In addition, our study demonstrated an indirect correlation between increasing age and survival $(\mathrm{p}<0.0001)$. There are many reports in the literature confirming that prognosis is consistently worsened with the increasing age of the patient at diagnosis $(13,14)$. This can partly be explained by more aggressive tumour biology (14), and partly by the associated co-morbidities which influence the choice of treatment in this group. Nevertheless, age alone should not influence treatment 
Table IX. Grade 3/4 morbidity associated with treatment (12 patients, 16 separate toxicities).

\begin{tabular}{|c|c|c|c|}
\hline Patient number & Treatment received & Morbidity/grade & Timing \\
\hline $4 *$ & $1 \mathrm{CT}+4000$ cGy $16 \#$ & 3D Bladder, $3 \mathrm{~A}$ rectum, $3 \mathrm{C}$ vagina & 7 years, 1 year \\
\hline $15 *$ & ICT x2, 5500 cGy & 3A Vagina & 2 years \\
\hline $25 *$ & ICT x2 7000 cGy & 3A Vagina & 5 years \\
\hline 38 & XRT only & Small bowel/4 & 2-3 months \\
\hline 39 & 4250 cGy $16 \#$, Vault Cs & Vesicocolic fistula/3 & 8 years \\
\hline 40 & ICT x2, 7125 cGy & 3B Sigmoid & 6 months \\
\hline 41 & ICT x 14650 cGy & 3B Small bowel & 1 year \\
\hline 42 & 4250 cGy $16 \#$, Vault Cs & 3 Vagina & 4 years \\
\hline 43 & 4000 cGy 20\# & 3 Sigmoid/obstruction & 7 years \\
\hline 44 & 4000 cGy 20\#, Sorbo Tx & 3A Vagina & 11 months \\
\hline 45 & 4000 cGy 20\#, Vault Cs & 3A Vagina & 3 years 6 months \\
\hline 46 & 4000 cGy $16 \#$, Vault Cs & 3C Vagina, 3A sigmoid & 13 months \\
\hline
\end{tabular}

Those patients marked with * appear in both Table VIII and IX.

choice. Whilst there may be an increase in the short-term mild/transient toxicity (15), we found no increase in late toxicity associated with radiotherapy in the older age group.

Whilst total abdominal hysterectomy and bilateral salpingoophorectomy is well recognised as the treatment of choice in stage I endometrial carcinoma, the value of postoperative radiotherapy is controversial. Although pelvic radiotherapy reduces vaginal and pelvic relapse $(18,19)$, distant metastases still occur in this group (noted in $84 \%$ of patients who relapsed in this survey), and no survival benefit has been confirmed. It is hoped that the ASTEC trial will help to establish whether the practice of giving adjuvant radiotherapy improves survival (unpublished data).

A study by Sartori et al in 2003, involving 209 patients with endometrial carcinoma (Stage I-IV), treated with surgery and/or XRT and/or chemotherapy, was undertaken to identify patterns of relapse, as well as factors influencing survival in those that relapse, and the outcome of salvage therapy (18). It demonstrated that most patients relapsed within 24 months. Adjuvant radiotherapy appeared to reduce the likelihood of pelvic recurrence in high-risk early stage disease (34.3 vs. $61.2 \%$ ), but seemingly the incidence of distant metastases (65.7 vs. $38.8 \%$ ) increased, presumably as more patients survived without local recurrence and then succumbed to distant metastases.

Therefore, whilst adjuvant radiotherapy reduces locoregional relapse, it has not been demonstrated to impact on overall survival. With this in mind Ackerman et al suggested that adjuvant therapy should be withheld and reserved for recurrence (19). Whilst they reported successful treatment in $67 \%$, the 5-year survival in this group was only $43 \%$. Similar findings were reported by Jereczek-Fossa et al, where, although the clinical tumour response at relapse was $73 \%$, the 5 -year survival was only $25 \%$, as $67 \%$ of responders developed progressive disease after a median of 9 months (20). Our data also demonstrate a poorer overall outcome in those treated at the time of relapse, with a 5-year survival of $53.6 \%$.
Since a substantial number of recurrences occur outside the pelvis the role of adjuvant chemotherapy has been explored, with some success $(21,22)$. Our data would support the need for adjuvant chemotherapy, with the majority of recurrent disease (84\%) occurring outside the pelvis. Further randomised studies are necessary to confirm or dispute the efficacy of adjuvant systemic treatment.

It is necessary to weigh serious complications of adjuvant radiation against the expected benefit of treatment. Weiss et al (23), explored this in a retrospective analysis of 159 patients with early stage (I and II) endometrial cancer, treated with XRT and brachytherapy following definitive surgery (TAH + BSO). Late toxicity was noted in $20.6 \%$. Most symptoms were mild or moderate, with severe symptoms (3 and/or 4) occurring in $2 \%$ of patients.

Due to a perceived increased risk of morbidity from postoperative XRT, we avoided XRT in 30 'frail' (increased age and co-morbidity) patients and elected to treat this group with brachytherapy alone. There was no significant difference in pelvic recurrence in this group or in overall survival compared with the other radically treated groups. The results of PORTEC 2 may clarify the indication for external beam and/or brachytherapy as adjuvant treatment (Creutzberg CL, et al, unpublished data). The benefits of vault brachytherapy over external beam treatment in the adjuvant setting has been demonstrated by others $(24,25)$.

The incidence of $3 \%$ severe late (grade 3 and/or 4 using the Franco-Italian glossary) treatment-related complications recorded in the XRT group found by Creutzberg et al (26) is consistent with our data $(3.8 \%)$, using the same scoring system. Most of these complications originated from the bowel. In our data set similar numbers affected the bowel and the vagina (7/16). Whilst vaginal stenosis may not be lifethreatening it is often burdensome for the woman and has the potential to profoundly affect her physical, psychological and social functioning and hence quality of life. The evidence suggest that compliance with dilator use is increased when a 
designated person provides specific instructions and information about use, and that poor compliance is related to insufficient or no information or lack of clarification (27).

Weiss et al noted that in many patients there is no symptom-free interval following radiotherapy (23). It has been argued that these complications should be regarded as consequential, arising as a result of severe or persisting acute toxicity. This may have accounted in part for the short survival of one patient during treatment in this series which occurred at 3 months as a result of small bowel injury.

There were a total of 49 intercurrent cases of patients succumbing in this cohort. This reflects the co-morbidity of patients with endometrial cancer, and is supported by the literature which confirms a frequent association between medical disorders and endometrial cancer $(28,29)$. Obesity is one of the most common disorders in medical practice. Its incidence is on the increase and it is a major public health problem. Obesity limits the ability to accurately stage the disease, a factor established as a prognostic factor for survival in this data set and others (30). Wang et al showed that $11 \%$ of patients with co-morbidity did not receive surgical treatment compared to $1.9 \%$ of controls, a result that is statistically significant $(\mathrm{p}<0.001)$, necessitating the use of radiotherapy (31). However, whilst co-morbidity influenced the selection of treatment strategy, Wang et al did not demonstrate any significant survival disadvantage. This contradicts the study of Sharma et al (32), and our data set, where the relationship between co-morbidity (represented by the ACE-27 score) and survival was highly significant $(\mathrm{p}<0.0005)$.

In conclusion, these data demonstrate that survival in patients with endometrial carcinoma treated with radical intent is good, with stage and grade of tumour being significant factors for overall survival. The incidence of locoregional recurrence was low across all treatment groups, with most patients in this group experiencing distant relapse. This supports a possible role for adjuvant chemotherapy.

The co-morbidity (ACE-27) score is significantly associated with poorer survival $(\mathrm{p}<0.0055)$ and increased late morbidity from adjuvant radiotherapy $(\mathrm{p}=0.0019)$. Increasing age was not a significant predictor of morbidity and as such, should not be used as a parameter for treatment selection.

\section{References}

1. Visser O, Coebergh JWW, Schouten LJ and Van Dijk JAAM: Incidence of cancer in the Netherlands 1995. Utrect: Netherlands Cancer Registry, 43, 1998.

2. Jereczek-Fossa BA, Badzio A and Jassem J: Surgery followed by radiotherapy in endometrial cancer: Analysis of survival and patterns of failure. Int J Gynecol Cancer 9: 285-294, 1999.

3. Roberts JA, Brunetto VL, Keys HM, et al: A phase III randomised study of surgery versus surgery plus adjuvant radiation therapy in intermediate-risk endometrial carcinoma (GOG 99). Soc Gynecol Oncol 99: 70, 1998.

4. Fishman D, Roberts K, Chambers J, et al: Radiation therapy as exclusive treatment for medically inoperable patients with stage I and II endometrial carcinoma. Gynecol Oncol 61:189-196, 1996.

5. Chassagne D, Sismondi P, Horiot JC, Sinistrero G, Bey P, Zola P, Pernot M, Gerbaulet A, Kunkler I and Michel G: A glossary for reporting complications of treatment in gynaecological cancers. Radiat Oncol 26: 195-202, 1993.

6. Charlson ME, Pompei P, Ales KL and MacKenzie CR: A new method for classifying prognostic comorbidity in longitudinal studies: development and validation. J Chron Dis 40: 373-383, 1987.
7. Linn BS, Linn MW and Gurel L: Cumulative illness rating scale. J Am Geriatr Soc 16: 622-626, 1968.

8. Greenfield S, Apolone G, McNeil BJ and Cleary PD: The importance of co-existent disease in the occurrence of postoperative complications and one year recovery in patients undergoing total hip replacement. Comorbidity and outcomes after hip replacement. Med Care 31: 141-154, 1993.

9. Kaplan MH and Feinstein AR: The importance of classifying initial co-morbidity in evaluating the outcome in diabetes mellitus. J Chron Dis 27: 387-404, 1974.

10. Peto R, Pike MC, Armitage P, Breslaw NE, Cox DR, Howard SV, et al: Design and analysis of randomised clinical trials requiring prolonged observation of each patient. II Analysis and examples. Br J Cancer 35: 1-39, 1977.

11. FIGO annual report on the results of treatment in gynaecological cancer. Int J Gynecol Obstet 83: 1-229, 2003.

12. Steiner E, Eicher O, Sagemuller J, Schmidt M, Pilch H, Tanner B, et al: Multivariate independent prognostic factors in endometrial carcinoma: A clinicopathologic study in 181 patients: 10 years experience at the Department of Obstetrics and Gynecology of the Mainz University. Int J Gynecol Cancer 13: 197-203, 2003.

13. Troung PT, Kader HA, Lucy B, Lesperance M, MacNeil MV, Berthelet E, et al: The effect of age and co-morbidity on treatment and outcome in women with endometrial cancer. Am J Clin Oncol 28: 157-164, 2005.

14. Alektiar KM, Venkatraman E, Abu-Rustum N, et al: Is endometrial carcinoma intrinsically more aggressive in elderly patients? Cancer 98: 2368-2377, 2003.

15. Jereczek-Fossa BA, Badzio A and Jassem J: Factors determining acute normal tissue reactions during postoperative radiotherapy in endometrial cancer: analysis of 317 consecutive cases. Radiat Oncol 68: 33-39, 2003.

16. Aalders J, Abeler V, Kolstad P and Onsrud M: Postoperative external irradiation and prognostic parameters in stage I endometrial carcinoma: clinical and histopathological study of 540 patients. Obstet Gynecol 56: 419-427, 1980.

17. Creutzberg CL, van Putten WL, Koper PC, et al: Surgery and postoperative radiotherapy versus surgery alone for patients with stage I endometrial carcinoma: Multicentre randomised trial-PORTEC study group, post operative radiation therapy in endometrial carcinoma. Lancet 355: 1404-1411, 2000.

18. Sartori E, Laface B, Gadducci A, Maggino T, Zola P, Landoni F and Zanagnolo V: Factor's influencing survival in endometrial cancer relapsing patients: a cooperation task force (CTF) study. Int J Gynecol Cancer 13: 458-465, 2003.

19. Ackerman I, Malone S, Thomas G, et al: Endometrial carcinoma: Relative effectiveness of adjuvant irradiation versus therapy reserved for relapse. Gynecol Oncol 60: 177-183, 1996.

20. Jereczek-Fossa BA, Badzio A and Jassem J: Recurrent endometrial cancer after surgery alone: results of salvage radiotherapy. Int J Radiat Oncol Biol Phys 48: 405-413, 2000.

21. Burke TW, Gerhenson DM, Morris M, Stringer CA, Levenback C, Tortolero-Luna G and Baker VV: Postoperative adjuvant cisplatin, doxorubicin, and cyclophosphamide (PAC) chemotherapy in women with high-risk endometrial carcinoma. Gynecol Oncol 55: 47-50, 1994 .

22. Aoki Y, Watanabe M, Amikura T, Obata H, Sekine M, Yahata T, et al: Adjuvant chemotherapy as treatment of high risk stage I and II endometrial cancer. Gynecol Oncol 94: 333-339, 2004.

23. Weiss E, Hirnle P, Arnold-Bofinger H, Hess CF and Bamberg M: Therapeutic outcome and relation of acute and late side effects in the adjuvant radiotherapy of endometrial stage I and II. Radiat Oncol 53: 37-44, 1999.

24. Rittenberg PVC, Lotocki RJ, Heywood MS, Jones KD and Krepart GV: High-risk surgical stage I endometrial cancer: outcomes with vault brachytherapy alone. Gynecol Oncol 89: 288-294, 2003.

25. Chadha M, Nanavati PJ, Liu P, Fanning J and Jacobs A Patterns of failure in endometrial carcinoma stage 1B grade 3 and IC patients treated with postoperative vaginal vault brachytherapy. Gynecol Oncol 75: 103-107, 1999.

26. Creutzberg CL, van Putten WLJ, Koper PC, Lybeert MLM, Jobsen JJ, Warlam-Rodenhuis CC, et al: The morbidity of treatment for patients with stage I endometrial cancer: results from a randomised trial. Int J Radiat Oncol Biol Phys 51: 1246-1255, 2001.

27. Lancaster L: Preventing vaginal stenosis after brachytherapy for gynaecological cancer: an overview of Australian practices. Eur J Oncol Nurs 8: 30-39, 2004. 
28. Soler M, Chatenoud L, Negri E, Parazzini F, Franceschi S and Vecchia CL: Hypertension and hormone-related neoplasm. Hypertension 34: 320-325, 1999.

29. Anderson KE, Anderson E, Mink PJ, et al: Diabetes and endometrial cancer in the Iowa women's health study. Cancer Epidemiol Biomarkers Prev 10: 611-616, 2001.

30. Mariani A, Webb MJ, Keeney GL, et al: Surgical stage I endometrial cancer: predictors of distant failure and death. Gynecol Oncol 87: 274, 2002.
31. Wang CJ, Leung SW, Chen HC, et al: The correlation of acute toxicity and late rectal injury in radiotherapy for cervical carcinoma: evidence suggestive of consequential late effect (CQLE). Int J Radiat Oncol Biol Phys 40: 85-91, 1998.

32. Sharma DN, Chander S, Gairola M, et al: Medical disorders associated with endometrial carcinoma. J Assoc Physicians India 49: 630-633, 2002. 